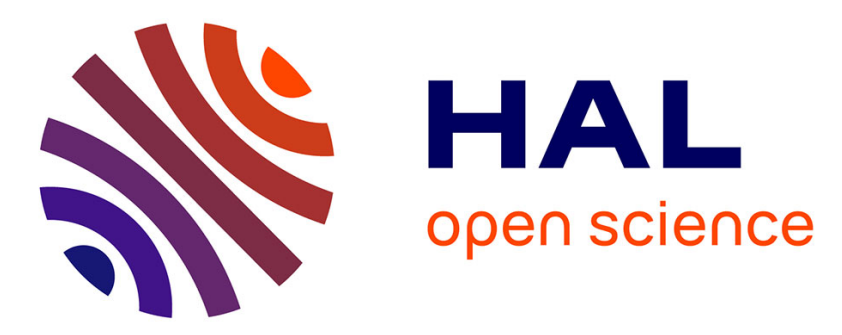

\title{
Du Danemark au Fossé rhénan. Un siècle d'analyse des voies de communication protohistoriques: évolution des méthodes et mise en commun des données
}

\author{
Loup Bernard, Rémy Wassong
}

\section{- To cite this version:}

Loup Bernard, Rémy Wassong. Du Danemark au Fossé rhénan. Un siècle d'analyse des voies de communication protohistoriques: évolution des méthodes et mise en commun des données. Archimède: archéologie et histoire ancienne, 2016, 3, pp.169-183. hal-01587264

\section{HAL Id: hal-01587264 \\ https://hal.science/hal-01587264}

Submitted on 13 Sep 2017

HAL is a multi-disciplinary open access archive for the deposit and dissemination of scientific research documents, whether they are published or not. The documents may come from teaching and research institutions in France or abroad, or from public or private research centers.
L'archive ouverte pluridisciplinaire HAL, est destinée au dépôt et à la diffusion de documents scientifiques de niveau recherche, publiés ou non, émanant des établissements d'enseignement et de recherche français ou étrangers, des laboratoires publics ou privés. 


\section{ARCHIMÈDE N'3}

\section{DOSSIER THÉMATIQUE : DES FOSSÉS ET DES REMPARTS. ENCEINTES ET SITES FORTIFIÉS DU RHIN SUPÉRIEUR ENTRE PROTOHISTOIRE ET MOYEN ÂGE}

\section{Olivier BUCHSENSCHUTZ}

Avant-propos. Des enceintes en terre anhistoriques à Google Earth

8 Lizzie SCHOLTUS

Histoire de la recherche dans le bassin de Saint-Dié-des-Vosges

20 Maxime WALTER

Les sites de hauteur du massif vosgien. Actualisation des données et modalités d'implantation

37 Jean-Jacques SCHWIEN

Chateaux et enceintes des Vosges du Nord. Topographie et longue durée

49 Anne-Marie ADAM

La palissade dans tous ses états : I'enclos du Britzgyberg (Illfurth, Haut-Rhin) et autres aménagements palissadés dans les habitats du premier âge du Fer

60 Clément FÉLIU

L'enceinte inférieure du Frankenbourg (67) et les remparts à poteaux frontaux de la fin de l'âge du Fer dans l'espace du Rhin supérieur. Pour une révision de la typologie des Pfostenschlitzmauern

74 Jacky KOCH et Thomas FISCHBACH

Enceintes de hauteur en pierres et formes « primitives » de châteaux ? L'exemple du Bernstein

87 Adrien VUILLEMIN

Les enceintes urbaines en moyenne Alsace (1200-1850)

102 Jean-François PININGRE

Les enceintes de l'âge du Bronze et du premier âge du Fer en Franche-Comté. Un bilan des recherches

124 Clément FÉLIU et Jean-Jacques SCHWIEN

Conclusion. Nouvelles perspectives sur les enceintes du Rhin supérieur

\section{ACTUALITÉ DE LA RECHERCHE : ARCHÉOLOGIE DES RÉSEAUX}

\section{Claire CAMBERLEIN}

Les réseaux en archéologie : approche historiographique et interdisciplinaire

135 Thomas HUTIN

Lieux d'échanges et espaces publics en Gaule à La Tène finale

150 Streeve GENTNER

Économie du fer et voies de communication, de l'abattage du minerai à la distribution

du métal : I'exemple du nord de la Forêt-Noire au Ve siècle av. J.-C.

169 Loup BERNARD et Rémy WASSONG

Du Danemark au Fossé rhénan. Un siècle d'analyse des voies de communications protohistoriques : évolution des méthodes et mise en commun des données

184 Steeve GENTNER et Rémy WASSONG

Conclusion. L'archéologie des réseaux : une thématique aux multiples facettes

\section{VARIA}

187 Fábio VERGARA CERQUEIRA

To march in phalanx, to jump with weights, to tread the grapes, to knead the bread. What is the aulos for?

206 Hermann AMON

Les supra-commandements comme solution à la crise militaire du III siècle de l'Empire romain sous Philippe l'Arabe et Gallien

218 Martina BONO

Il processo di Cremuzio Cordo in Dio LVII, 24, 2-4

\section{LA CHRONIQUE D'ARCHIMÈDE}

228 Frédéric COLIN (éd.)

La Chronique d'Archimède. Bilan des activités scientifiques 2015-2016 de I'unité mixte de recherche 7044 


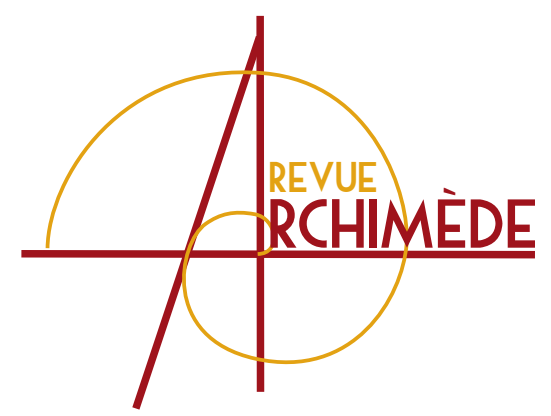

\section{DU DANEMARK AU FOSSÉ RHÉNAN. UN SIÈCLE D'ANALYSE DES VOIES DE COMMUNICATION PROTOHISTORIQUES : ÉVOLUTION DES MÉTHODES ET MISE EN COMMUN DES DONNÉES}

\author{
Loup BERNARD \\ Maître de conférences \\ Université de Strasbourg \\ UMR 7044 Archimède \\ loup.bernard@unistra.fr
}

\author{
Rémy WASSONG \\ Doctorant \\ Université de Strasbourg \\ UMR 7044 Archimède \\ remy.wassong@hotmail.fr
}

\section{RÉSUMÉ}

La perception des réseaux viaires protohistoriques suscite un vif intérêt dans la recherche actuelle. Dès le début $\mathrm{du} \mathrm{xx}^{\mathrm{e}}$ siècle, les chercheurs ont formulé des hypothèses concernant un lien supposé entre les zones funéraires et les axes de communication et ont tenté de les cartographier. Cet article propose de passer en revue, à titre d'exemple, les études de Sophus O. Müller au Danemark et de Xavier Nessel dans la forêt de Haguenau et leurs mises à jour. Les auteurs soulignent l'évolution des méthodes de cartographie des axes de communication protohistoriques en relevant les apports et les limites des techniques actuelles

ArkeoGIS. et de la mise en commun des données grâce, entre autres, au logiciel ArkeoGIS.
Since the beginning of the $\mathrm{xx}^{\text {th }}$ century, scholars tend to apprehend and map the protohistoric pathway networks. This paper presents the early work of Sophus O. Müller in Denmark and Xavier Nessel in the wood of Haguenau with their updated datas. The authors emphasize the evolution of ancient pathway mapping methods and show the contributions and limits of modern techniques and data pooling thanks to some softwares such as ArkeoGIS.
KeYWORDS Geographical Information System, tumulus, ancient pathway, Haguenau, Hallstatt, ArkeoGIS. 
L'absence de traces archéologiques rend difficile la perception des réseaux viaires protohistoriques. Dès le début du $x x^{e}$ siècle, quelques chercheurs ont formulé des hypothèses concernant le lien entre les zones funéraires et les axes de communication et ont tenté de les cartographier. Ainsi, les études de Sophus O. Müller, au Danemark, et celles de Xavier Nessel, dans la forêt de Haguenau, ont fait l'objet de reprises récentes dans le cadre de travaux universitaires qui ont permis d'affiner, de compléter et de mettre à jour les données. En recoupant les données archéologiques et textuelles, et en mettant en commun un grand nombre de bases de données, nous sommes en mesure, avec des moyens actuels comme ArkeoGIS, de proposer et de modéliser des hypothèses plus pertinentes dans ce domaine malgré le caractère « impossible » de ce type de cartographie.

\section{ETUDES MICRORÉGIONALES : DES NÉCROPOLES TUMULAIRES DANOISES AUX COMPLEXES FUNÉRAIRES DE HAGUENAU}

\section{UN PRÉCURSEUR DANOIS}

En 1904, Sophus O. Müller, archéologue et directeur du musée national danois de 1895 à 1921, publie un article mettant pour la première fois en relation l'implantation des nécropoles tumulaires avec les réseaux de communication anciens [1]. Il prend des clichés photographiques des nécropoles tumulaires danoises connues et procède à leur cartographie en mettant, dans un souci de diffusion au plus grand nombre, les échelles en mètres, trois ans avant la mise en place du système métrique au Danemark. L'auteur insiste sur le fait que la recherche se concentre principalement sur I'habitat et les tertres funéraires et néglige la compréhension des liens spatiaux qui les unissent. Sophus O. Müller met en avant le développement des tertres le long d'axes linéaires (déjà évoqué dans son ouvrage de 1897 [2]) et suggère la présence de «pis-

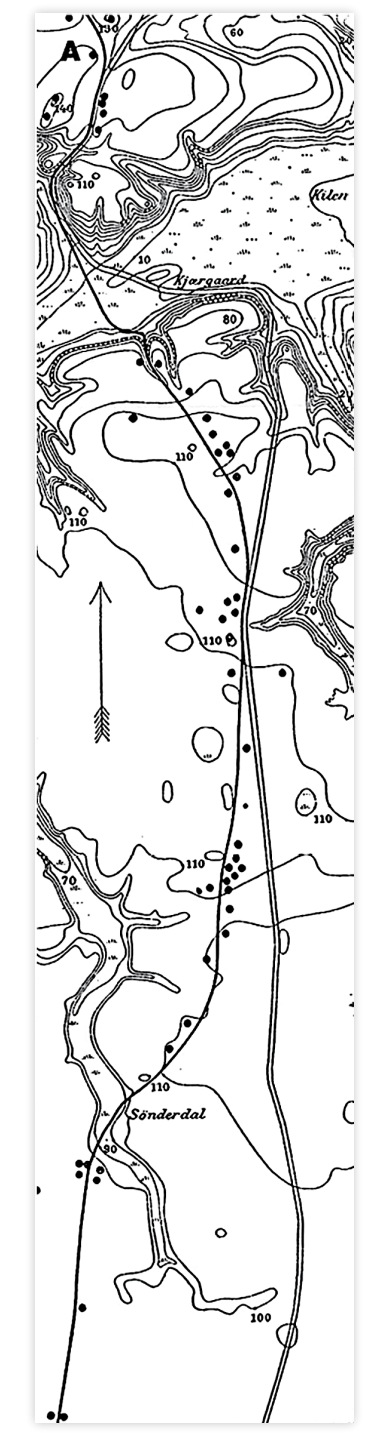

Figure 1

Cartographie d'une nécropole tumulaire, d'après MÜLleR 1904, p. 26. tes bien préparées » à proximité. Il voit bien que I'implantation des nécropoles tumulaires présente un lien particulier avec les formes du paysage naturel et constate la présence de groupes tumulaires au niveau des cols et de part et d'autre des cours d'eau, à des endroits où le passage semble assez aisé au moment où lui-même écrit son ouvrage. À la suite de cela, il propose l'existence de gués à ces emplacements dès les périodes préhistoriques. Les plus grands tertres de son étude (environ 50 mètres de diamètre) sont implantés en ces lieux remarquables. Il parvient également à identifier des carrefours et propose de restituer un véritable « réseau routier » (vejnettet) à l'intérieur duquel les habitats contemporains des tertres sont bel et bien intégrés, malgré la faible portion d'habitats suffisamment bien documentés à I'époque (fig. 1). Il faudra attendre les travaux de Lutz Klassen sur les habitats fortifiés datant du Néolithique à la période Viking [3] pour avoir accès à une meilleure connaissance de ces sites, et pour confirmer leur intégration dans les axes de communication présentés par Sophus O. Müller (fig. 2). Ce dernier propose la persistance de ce réseau du Néolithique à l'âge du Fer à travers les différentes périodes chronologiques représentées dans les tumuli. Sur les 7500 tertres qu'il a pu répertorier, seuls 390 sont datés.

[1] MÜLLER 1904, p. 1-64.

[2] MüLLER 1897, p. 298-301.

[3] KLASSEn 2014, p. 58-83. 
Ainsi 170 tertres sont occupés au cours du Néolithique (3900 à 1800 avant notre ère dans cette zone géographique), 140 à l'âge du Bronze (1800 à 500 avant notre ère), 60 tertres supplémentaires ont livré des sépultures de ces deux périodes et enfin 20 tertres sont datés de I'âge du Fer (500 avant notre ère à 1066 de notre ère, ou jusqu'au Ix ${ }^{e}$ siècle en excluant la période viking), auxquels il ajoute quatre sépultures plates découvertes fortuitement et qui s'intègrent parfaitement dans le réseau qu'il présente.

Sophus O. Müller ne dira jamais sur quoi se fonde son « intuition » pour la restitution du réseau routier préet protohistorique danois. Il paraît pourtant clair qu'il doit avoir à l'esprit les fouilles de Bror Emil Hildebrand à Gamla Uppsala en 1846, pour le tumulus est, et celles de 1874, pour le tumulus ouest [4] (fig. 3). Les résultats de ces fouilles ont en effet été largement diffusés. Ces tertres, datant de l'âge du Fer et de la période Viking, sont directement mis en lien avec divers textes portant sur des rites païens et avec les sagas scandinaves qui décrivent fréquemment les tertres et le choix de leur implantation (les recherches plus récentes montrent que les zones funéraires de Gamla Uppsala étaient utilisées dès l'âge du Bronze [5]). Nous ne reviendrons pas ici sur ces nombreuses occurrences [6], mais citons les vers les plus représentatifs du célèbre poème épique
Beowulf. Agonisant, le héros Beowulf dicte ses dernières volontés : «Que mes guerriers érigent un tumulus après avoir brûlé mon corps, ce monument témoignera de notre gloire! Que ce tertre, bien haut, s'élève sur le promontoire de Hrones, afin que les voyageurs et les marins l'aperçoivent de loin quand ils navigueront sur les flots ténébreux, et tous salueront le tertre de Beowulf [7] ». Sophus O. Müller n'établira jamais de liens entre ses constats archéologiques et ces sources littéraires, mais il ne fait aucun doute qu'il les connaît, la fin du $\mathrm{XIX}^{\mathrm{e}}$ et le début du $\mathrm{XX}^{\mathrm{e}}$ siècle étant marqués par la glorification du passé national, au Danemark comme dans une grande partie des pays européens de l'époque. Il est donc probable que les hypothèses émises par Sophus $O$. Müller puisent leur origine dans la littérature scandinave ancienne.

\section{UN RAISONNEMENT SIMILAIRE À HAGUENAU ?}

Pendant que le chercheur danois travaillait sur les axes de communication pré- et protohistoriques de sa patrie, le maire de Haguenau, Xavier Nessel, s'attaquait quant à lui à l'étude des nécropoles tumulaires de la forêt homonyme. En raison de son ampleur (près de 21000 hectares, cf. infra), le massif forestier de Haguenau (Bas-Rhin) a permis la préservation de nombreux sites archéologiques

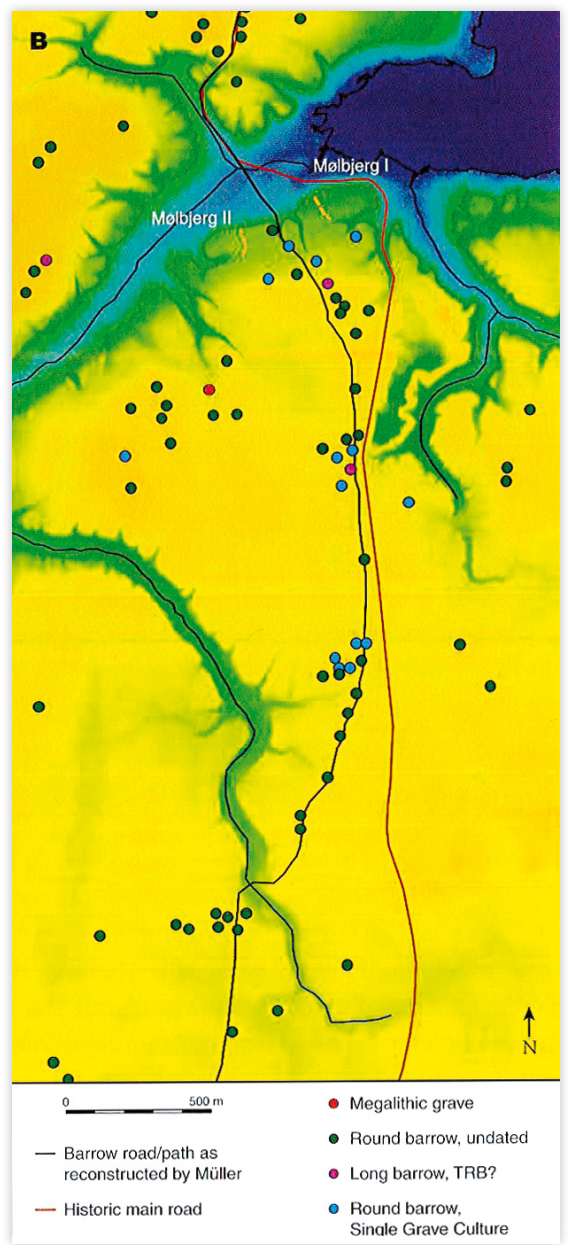

[4] LJUNGKVIST 2013, p. 33-68.

[5] FRÖLUND 2009.

[6] WASSONG 2013, p. 64-69.

[7] Beowulf, v. 2802 à 2808 (éd. et trad. Crépin, 2007).

< Figure 2 : reprise de la carte de S. O. Müller par Lutz Klassen avec la matérialisation des habitats, d'après KLASSEN 2014, p. 64.

$\checkmark$ Figure 3 : photographie des fouilles de Bror Emil Hildebrand sur un des tertres de Gamla Uppsala (Uppsala universitets samlingar).

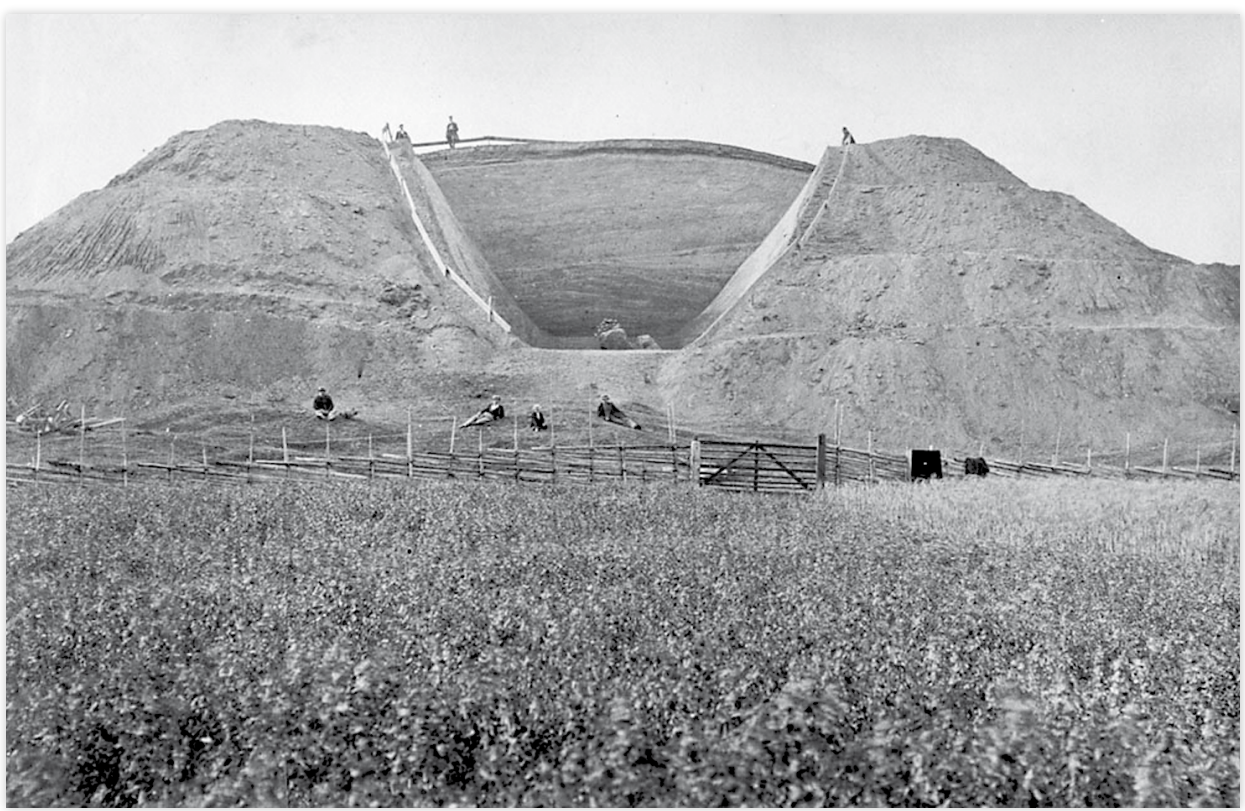




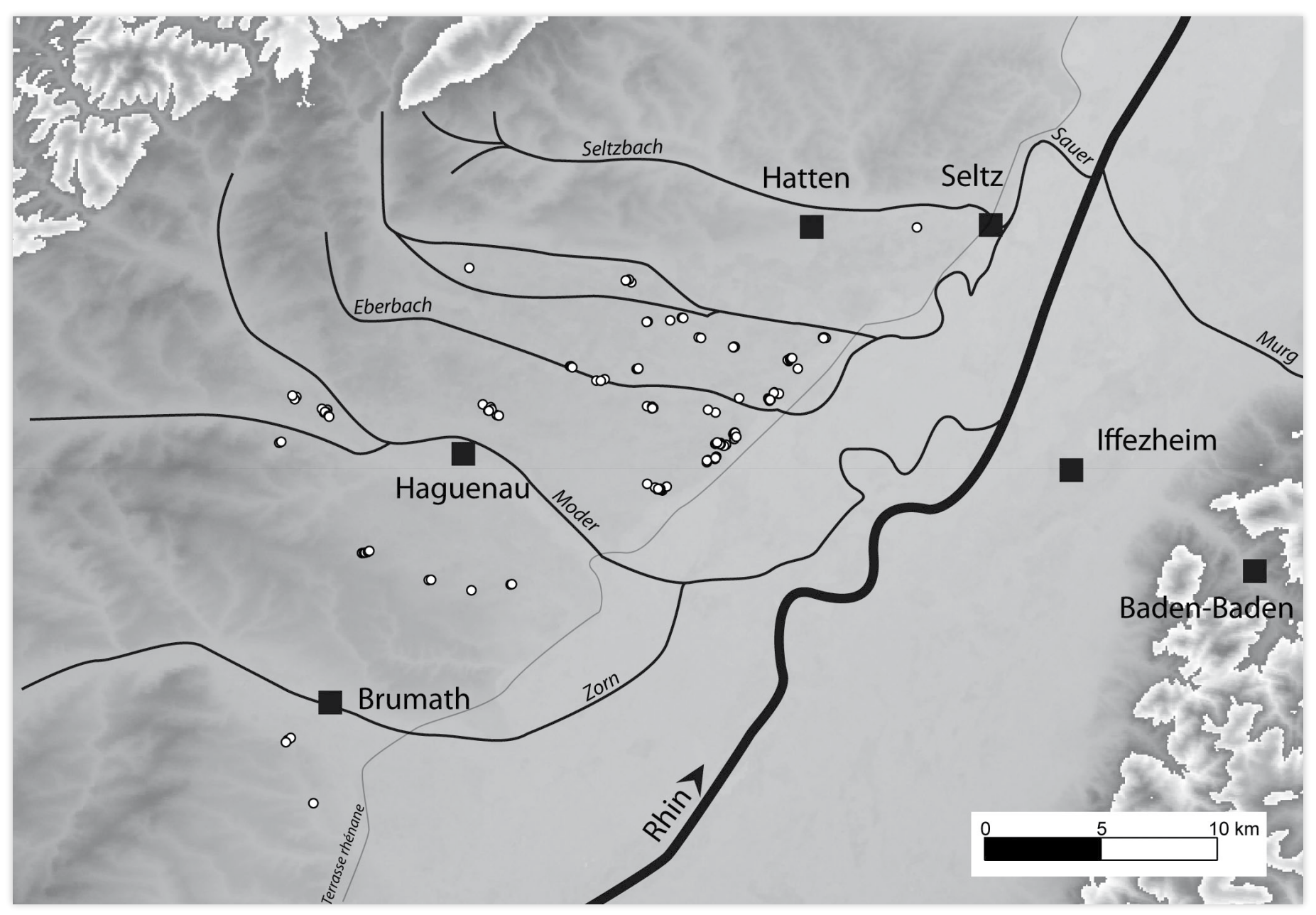

Figure 4

Les tertres occupés à l'âge du Bronze, d'après WASsONG 2015, modifié.

au cours du temps. À la suite de Maximilien de Ring, Robert Henning ou Ernst Wagner, Xavier Nessel procède à I'ouverture de plusieurs tertres à partir de 1862 . Il ne publiera toutefois jamais lui-même les données issues de ses recherches, et il faudra attendre l'ouvrage de Naue [8], publié en 1905, pour avoir un premier aperçu de la « collection Nessel » et surtout les publications de Claude Schaeffer [9]. D'abord axées sur le mobilier, ce n'est que bien plus tard que les recherches s'orienteront sur l'aspect spatial et l'organisation des nécropoles. Jean-Pierre Legendre est le premier à faire une analyse de l'évolution chronologique et spatiale d'une nécropole tumulaire : celle de Maegstub, située à l'extrême ouest du massif forestier [10]. En 2009 et 2010, une équipe du Pôle Archéologique Interdépartemental Rhénan, dirigée par Frank Abert, fait de même avec les nécropoles de Kurzgelaend, Schirrheinerweg [11] et Brumath [12]. C'est dans la continuité de ces travaux que nous nous sommes inscrits lorsque nous avons commencé à relever les tumuli des nécropoles restantes en 2010. Les premières études spatiales ont mis en évidence des alignements de tertres le long d'axes dont la chronologie couvre les âges du Bronze et du Fer. Il s'agissait dès lors d'étendre ces études aux autres nécropoles pour en déduire des relations intra-nécropole et inter-nécropoles au cours de la protohistoire. Notre raisonnement s'appuie sur 190 tertres dont la datation est assurée ; cela peut paraître peu au vu des 639 tertres répertoriés lors des phases de terrain, mais il est très difficile d'obtenir un échantillon plus représentatif (nous avons ici environ $30 \%$ de tertres datés; en son temps, Sophus O. Müller n'en avait que $5 \%$ ). Cet écart s'explique par la difficulté à réattribuer les tertres repérés sur le terrain aux données anciennes de Xavier Nessel ou des autres chercheurs (durant les recherches sur le terrain il est apparu qu'une bonne partie des cartes publiées par Claude Schaeffer comportaient de nombreuses erreurs : mauvaise orientation géographique, échelle erronée, distorsion du fond de carte), la disparition de plusieurs tertres depuis les

[8] NAUE 1905.

[9] SCHAEFFER 1926 et 1930.

[10] LEGENDRE 1989.

[11] АвERT 2009.

[12] Abert et Rieth 2010. Travail inédit, la base est disponible sur http://arkeogis.org/. 
excursions forestières de l'ancien maire de Haguenau, ou encore par l'absence de mobilier accompagnant les sépultures. En effet, Xavier Nessel a, dans de nombreux cas, remarqué l'absence d'offrandes funéraires, ce qui exclut toute possibilité de datation des tombes.

Les premiers tertres sont édifiés au cours de l'âge du Bronze (fig. 4). Les plus anciens tumuli datent du Bronze ancien. Seuls deux monuments sont attribuables à cette période dans notre zone d'étude. Il est difficile de dégager une quelconque appartenance à une nécropole ou bien de définir exactement les raisons de l'emplacement des tertres à partir de ces données. Il faut toutefois remarquer que ces deux tertres sont implantés le long de la terrasse rhénane. Il s'agit du seul relief présent dans la région. Celui-ci se caractérise par un dénivelé de près de 10 mètres au-dessus du Ried (région naturelle de prés inondables et de marécages). Durant le Bronze moyen, on assiste à la construction d'un grand nombre de tertres funéraires. C'est également à cette période qu'on retrouve le plus grand nombre de zones funéraires actives. Celles-ci se développent de façon régulière sur l'ensemble de la zone d'étude et forment de véritables nécropoles au sens strict du terme. Notons également que la zone la plus densément occupée correspond au bord de la terrasse rhénane. Dans les autres cas, les monuments sont généralement implantés à proximité des cours d'eau. De nouveaux tertres sont construits à proximité des tumuli datant du Bronze ancien. S'agit-il ici d'un rapprochement vis-à-vis de la tombe d'un ancêtre ou d'une simple continuité d'utilisation de la zone funéraire ? Il est difficile de déterminer une telle relation à partir des données dont nous disposons, mais une chose est certaine, ces tertres plus anciens ne constituent pas le noyau de développement de ces nécropoles, mais tout au plus un point de repère pour la zone funéraire. On constate parfois l'édification de tumuli autour de tertres plus anciens, ce qui conduit à une nécropole de forme circulaire, mais ce cas est rare en forêt de Haguenau. Au Bronze final, on assiste à une diminution de l'utilisation de la sépulture sous tumulus (seuls 16 tertres présentent une occupation datant de cette phase contrastant ainsi avec les 71 tumuli occupés au Bronze moyen). On observe principalement des réutilisations des tertres construits au cours du Bronze moyen. Cependant quelques tertres sont fondés au cours de cette période. Ces derniers s'intègrent alors dans les zones funéraires préexistantes et définies au cours du Bronze moyen.

Au début de l'âge du Fer (fig. 5), les pratiques funéraires s'inscrivent dans la continuité de celles du Bronze final. Seuls 10 tertres sont utilisés au cours de cette période, parmi lesquels deux monuments édifiés ex nihilo à proximité de tertres du Bronze moyen. Neuf de ces tumuli se situent le long de la terrasse rhénane. La période du Hallstatt $D$ présente un nouvel essor de construction et de réutilisation de la sépulture sous tumulus puisque 117 tertres sont occupés au cours de cette période. Cette dynamique sera identique au cours de La Tène ancienne. La plupart des zones funéraires définies dès le Bronze moyen est réutilisée, exception faite de celles qui se situent au cœur de la région étudiée. La plupart des sépultures sont intégrées dans des tertres préexistants, mais on assiste également à l'édification de tertres

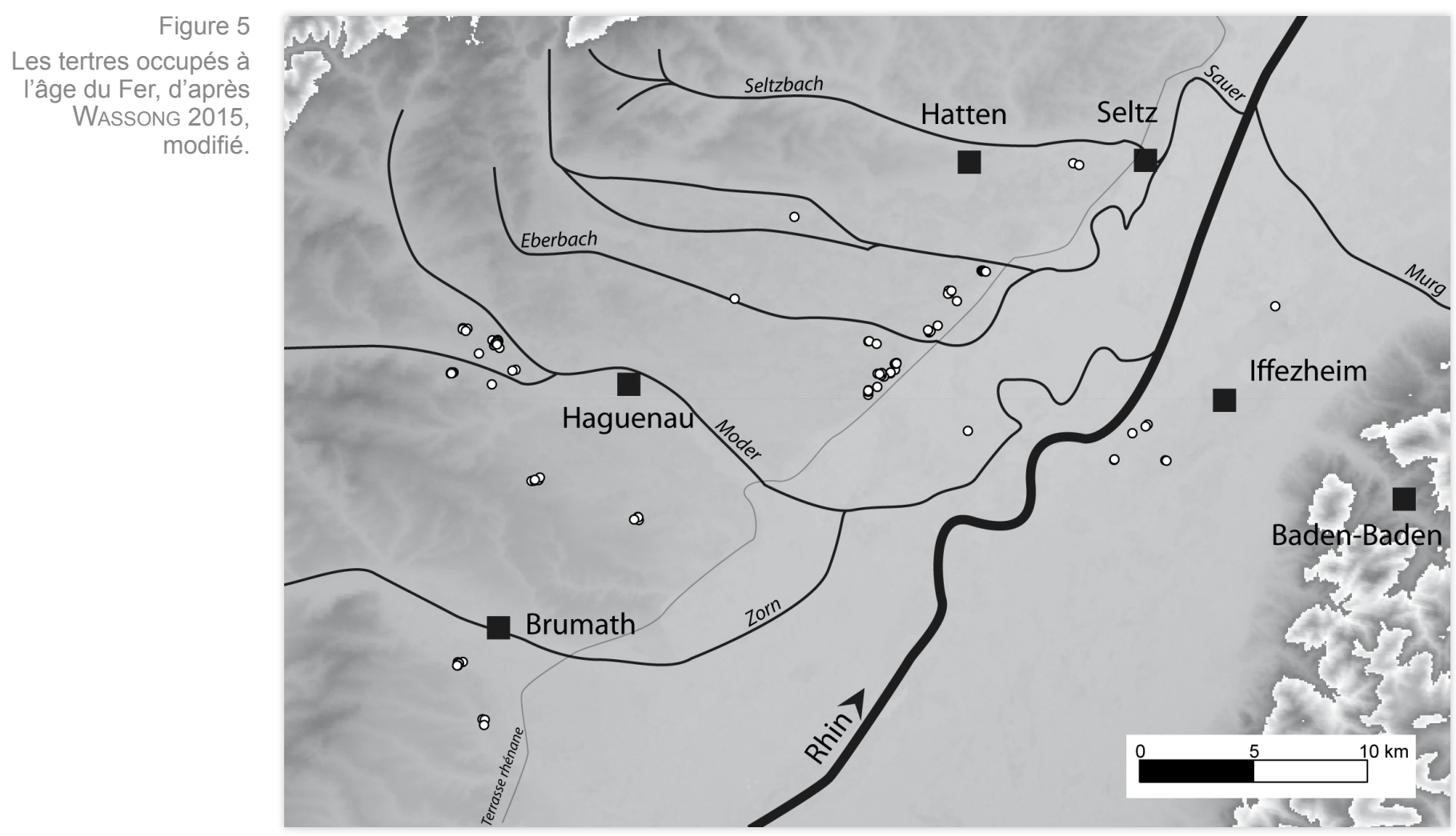


monumentaux, parfois isolés ou rassemblés au sein de petites nécropoles (le Goethehübel à Sessenheim ou encore les tumuli de Hügelsheim, par exemple). Si, comme nous le disions, les zones funéraires situées au centre du massif forestier ne sont plus utilisées, sauf quelques exceptions, c'est au profit de trois zones, dont deux n'ont jusqu'ici pas fait l'objet d'utilisation régulière, la dernière étant encore une fois la bordure de la terrasse rhénane. La première se situe à l'extrême ouest du massif forestier : bien qu'elle ait été déjà occupée dès le Bronze moyen, c'est véritablement au cours du Hallstatt $D$ et de La Tène ancienne que les nécropoles tumulaires qui s'y trouvaient prennent de l'importance. La seconde se situe sur la rive droite du Rhin et constitue une nouveauté. D'après l'état de nos connaissances actuelles, il s'agit des premières zones funéraires nouvellement définies depuis le Bronze moyen. Il semblerait en effet qu'aucun tertre antérieur au Hallstatt D n'ait été édifié sur la rive droite du Rhin.

Pour résumer, la plupart des zones funéraires et des nécropoles occupées au sein de notre secteur d'étude a été définie dès le Bronze moyen. Un nouvel élan dans I'utilisation de ce système funéraire est perceptible au Hallstatt $D$ et à La Tène ancienne, avant son arrêt quasi définitif à partir de 260 avant notre ère. Il est vrai que quelques objets datant de la période romaine ont été découverts dans des tertres par Maximilien de Ring. L'auteur des découvertes affirme que ces monuments ont probablement subi des perturbations postérieures en relation avec le dépôt de ces objets. Afin de ne pas subir ce biais, nous n'avons pas pris en compte ces tumuli.
Du point de vue spatial, on observe la prédominance de l'utilisation de la bordure de la terrasse rhénane au cours du temps. Les nécropoles sont parfois également alignées le long ou à proximité des cours d'eau qui parcourent le massif forestier, avec des espacements assez réguliers. Nous pouvons donc poser la question de l'existence d'un territoire organisé, voire normé.

\section{TUMULI, PAYSAGE ET RÉSEAU DE COMMUNICATION HIÉRARCHISÉ}

Au cours des recherches sur le terrain, nous avons pu remarquer l'étroite relation entre le paysage anthropique, qui se manifeste par les tertres, et les données naturelles du terrain. Cette relation se matérialise par le positionnement des tumuli à proximité de lieux remarquables, phénomène que Wolfgang Kimmig avait déjà mentionné pour les abords du site de la Heuneburg [13]. Dans notre cas, les emplacements remarquables du paysage se limitent aux cours d'eau et à leurs terrasses, parmi lesquelles la plus impressionnante est la terrasse rhénane dont l'élévation, que nous avons déjà évoquée auparavant, constitue un important lieu d'observation sur le Ried, mais aussi sur le Rhin lui-même et ses abords immédiats (fig. 6). Inversement, cette élévation est visible depuis le Ried à partir du moment où le couvert forestier n'est pas trop dense. La présence d'un tertre funéraire sur les rebords de cette terrasse n'est donc pas sans rappeler le

[13] Kimmig 1983.

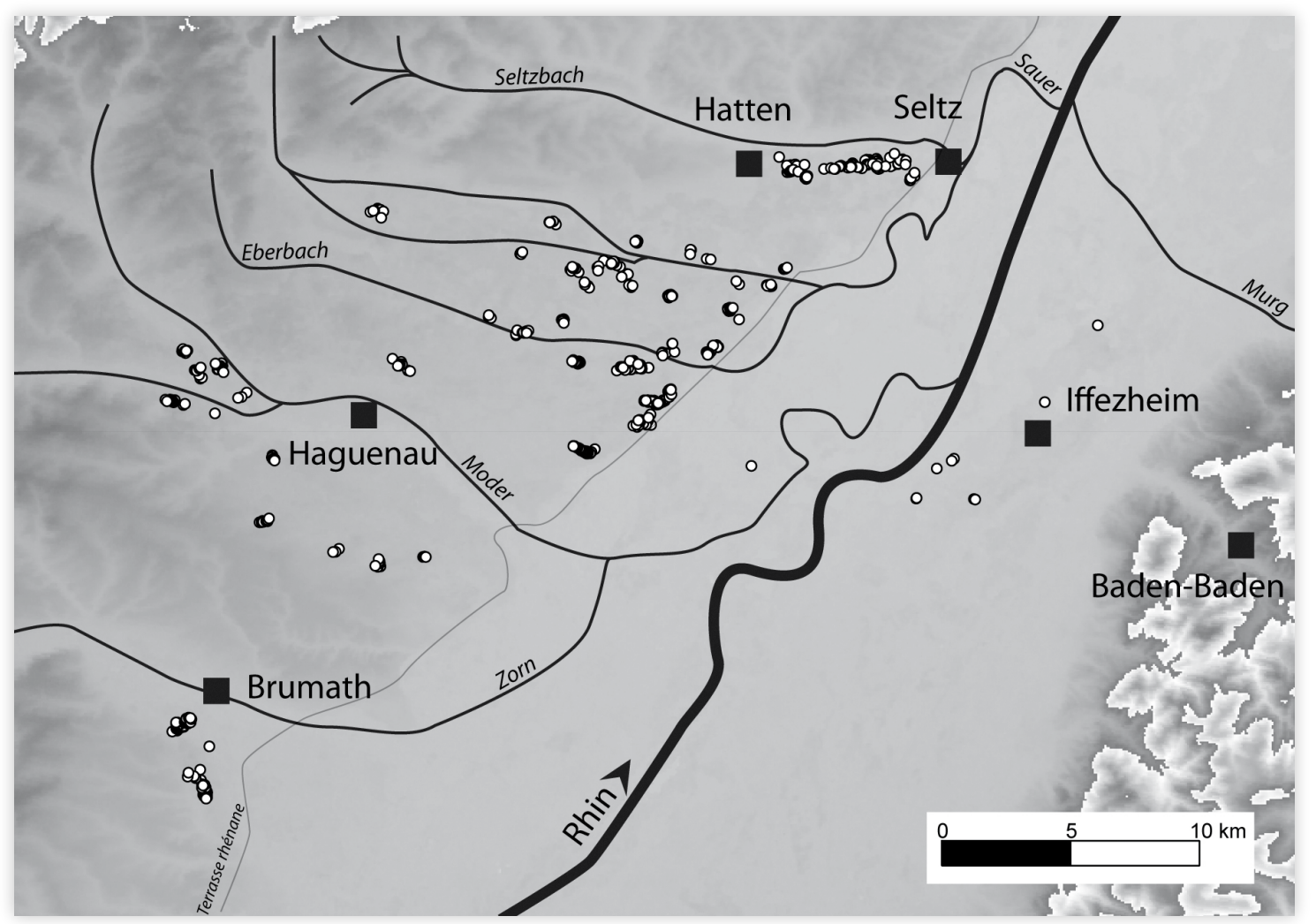

Figure 6

Ensemble des tertres relevés dans le secteur de Haguenau, d'après WASSONG 2015, modifié. 


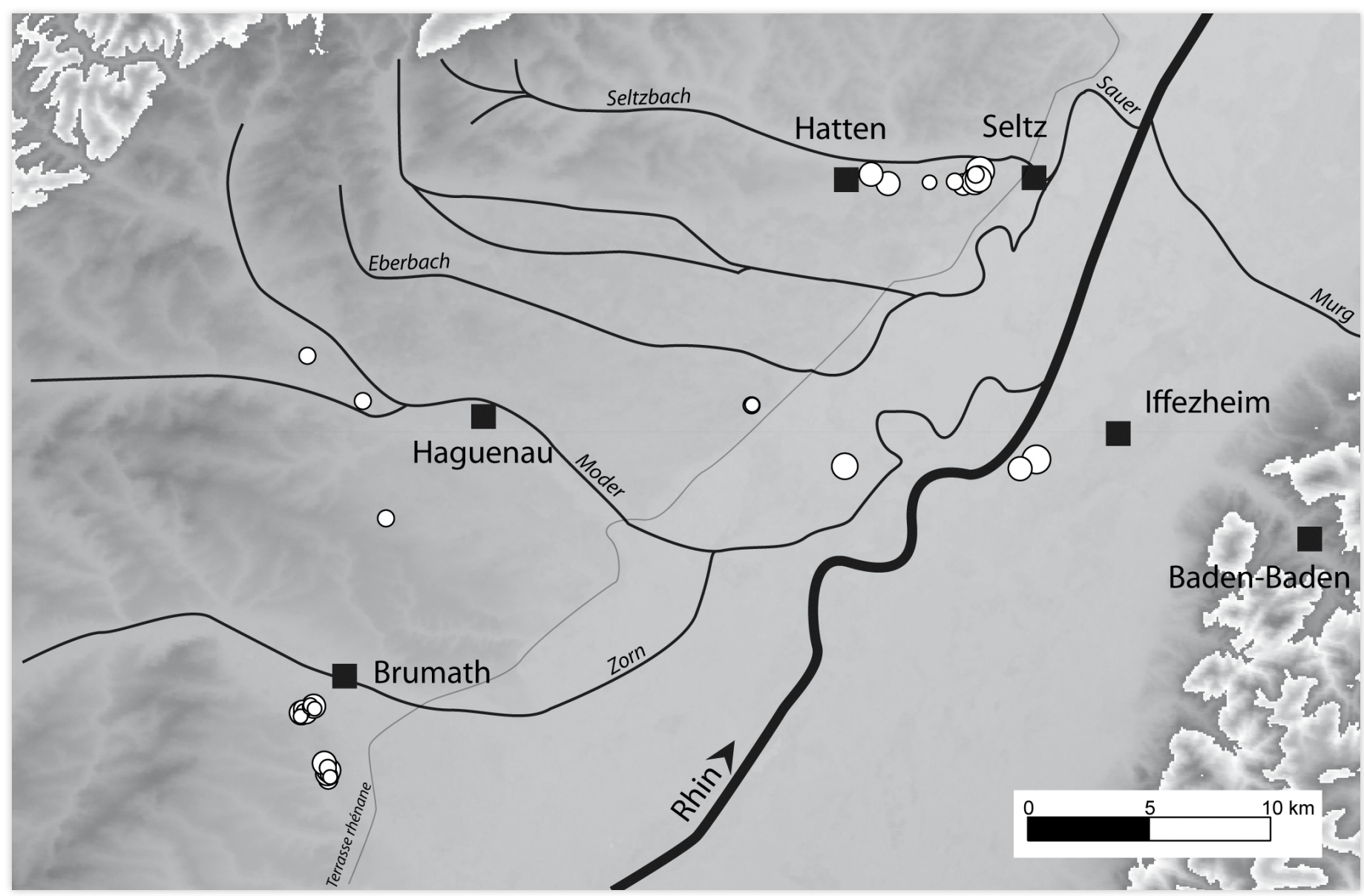

Figure 7

Emplacements des tertres de très grands volumes conservés, d'après WASSONG 2015, modifié.

positionnement du tumulus du héros Beowulf sur un promontoire, même s'il est délicat d'affirmer que les tertres rhénans remplissaient la même fonction. Il peut en être de même pour les tertres longeant les cours d'eau plus modestes de la région. Ces rivières fournissent généralement, si ce n'est un repère géographique constant, du moins un moyen de déplacement et de transport efficace à partir du moment où elles sont entretenues. Dans cette optique, il faut remarquer l'important axe nord-sud que constituent le Rhin et ses terrasses : c'est à proximité de cet axe que les zones funéraires les plus vastes et les tertres les plus volumineux ont été observés (fig. 7).

À l'âge du Bronze, le long de cours d'eau comme l'Ill et le Rhin, et de part et d'autre de leurs lits, des dépôts ont été effectués à intervalles réguliers [14]. Thierry Logel fait de ces endroits des « lieux privilégiés » pour les dépôts d'objets de prestige et propose, en recoupant ces données avec les anciens lits des cours d'eau, des emplacements pour les gués au cours de l'âge du Bronze. Deux d'entre eux se situent dans notre zone d'étude : le premier entre les villes de Fort-Louis et Hügelsheim, et le second entre Seltz et Rastatt. Durant I'âge du Fer, ce sont ces mêmes secteurs de dépôt qui accueillent désormais des tertres funéraires. S'il existe bien un lien entre ces lieux de passage et les dépôts à I'âge du Bronze, la continuité d'utilisation de ces zones dans le but d'y implanter des sépultures, parfois monumentales, tend à montrer que le lien constaté par Thierry Logel perdure au cours des périodes suivantes. Nous savons également que les zones de gué ne changent que très peu au fil du temps, comme I'a par exemple démontré Annie Dumont pour les gués de la Grande Saône dont les emplacements ne varient pas entre la protohistoire et l'époque moderne [15].

Les tertres funéraires seraient donc bien en relation avec des lieux de passage qui sont définis au départ par des données géographiques naturelles (gués, terrasses alluviales, élévations naturelles du terrain, cours d'eau). Ces espaces privilégiés sont également repris par la suite pour implanter des voies de communication aménagées dès la période romaine. C'est en particulier le cas des routes départementales 28 et 37 qui, sur une bonne partie de leur trajet, suivent les voies antiques et médiévales (fig. 11, infra). La première relie les villages de Hatten et de Seltz ; la seconde, quant à elle, relie Brumath à Seltz.

[14] LOGEL 2007.

[15] DUMONT 2002. 


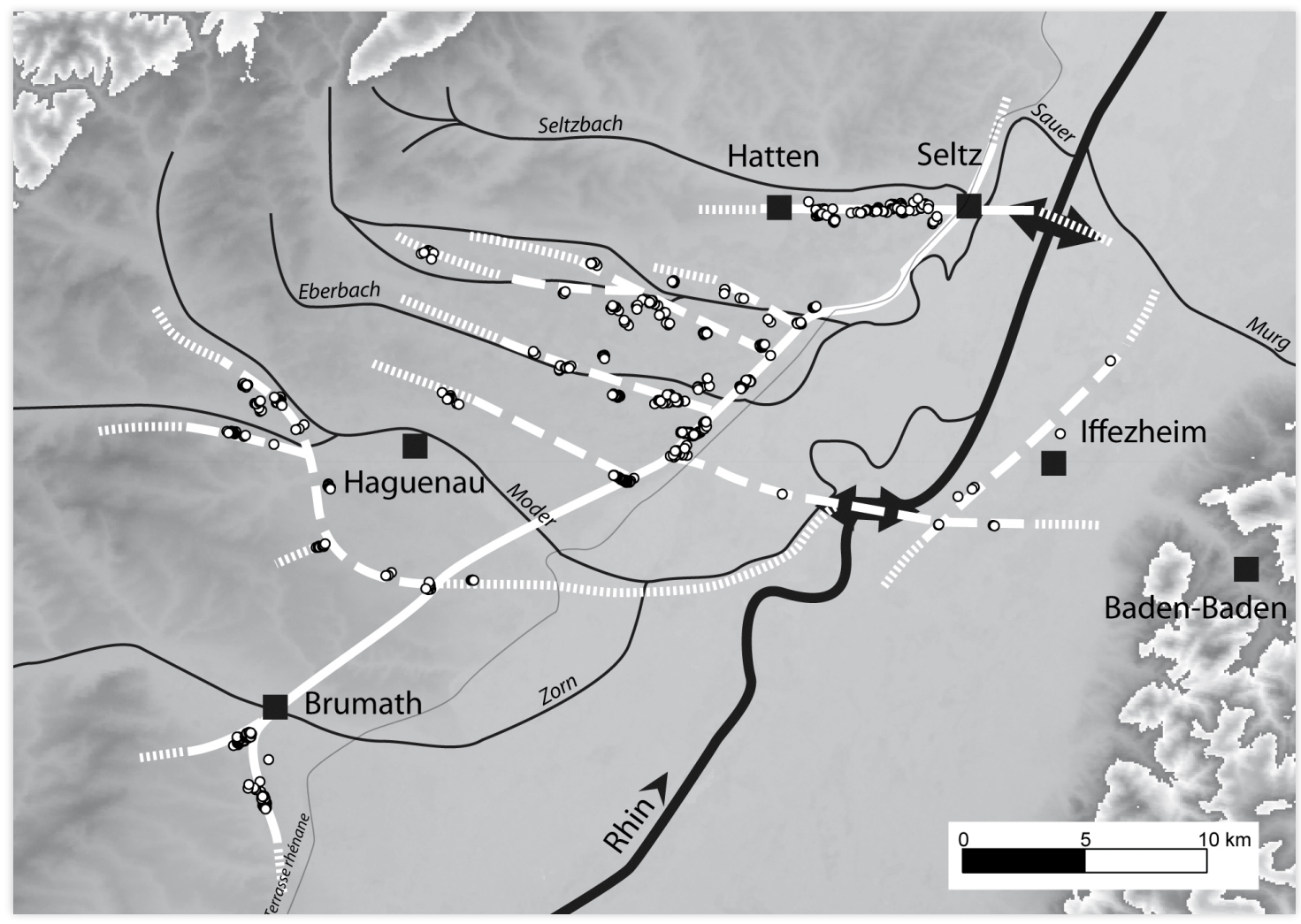

Figure 8

Hypothèse de restitution du réseau de communication protohistorique dans le secteur de Haguenau, d'après WASSONG 2015, modifié. En trait plein, les axes majeurs; en tirets, les axes secondaires; en pointillés, les prolongements possibles de ces axes.

Il semblerait donc que les tertres funéraires soient bel et bien installés le long d'axes de communication qui n'étaient peut-être qu'entretenus et non complètement aménagés à l'image des voies romaines. L'ensemble de ces données nous permet de proposer une hypothèse de restitution des voies de communication protohistoriques (fig. 8) [16].

Ce tissu viaire peut également être hiérarchisé. Nous I'avons vu, l'axe nord-sud semble jouer un rôle majeur dès le début de l'édification des tertres funéraires. Cette persistance au cours du temps et la densité des nécropoles qui le longent nous amènent à le qualifier d'axe principal, véritable épine dorsale du réseau. L'ensemble des autres voies dispose d'une moindre densité de monuments et d'une discontinuité d'occupation, ce qui n'exclut pas l'utilisation des voies aux périodes où les tertres ne sont pas utilisés ou construits. Ceci les relègue à un rang secondaire au sein de l'organisation territoriale. L'axe est-ouest au nord de la zone d'étude et reliant les villages de Hatten et Seltz joue vraisemblablement un rôle important comme l'attestent les réutilisations ultérieures (voies antiques et médiévales, route

[16] En l'absence d'autres éléments comme du parcellaire ou des habitats contemporains, notre réflexion doit se limiter à la «simple » étude des tertres, à l'inverse de ce qui a pu être fait dans d'autres régions pour d'autres périodes (Chevalier 2009 par exemple). Les aspects méthodologiques ou les méthodes régressives n'ont donc pas été appliquées ici, leur développement constituerait un article à part entière. départementale actuelle), et ce malgré l'absence de données chronologiques exploitables. Ces réutilisations plus tardives montrent bien qu'un coup d'arrêt dans I'utilisation de la zone funéraire n'implique pas I'abandon total d'un itinéraire.

Cette hiérarchisation des axes de circulation permet de proposer une hypothèse concernant les zones ne présentant aucun monument tumulaire. Outre la possibilité qu'il s'agisse d'emplacements réservés à des sépultures en tombes plates, et donc invisibles de nos jours, nous pouvons imaginer que l'éloignement par rapport à un axe majeur conditionne la diminution de l'utilisation des zones funéraires puis, par la suite, les secteurs de «vide». Plus le voyageur s'éloigne de l'axe directeur, moins les nécropoles sont denses et développées, jusqu'au moment où il se trouve trop éloigné de cette épine dorsale, en un lieu où aucune nécropole n'est établie. Ce serait donc aussi logiquement aux abords de ces axes majeurs que se situeraient les habitats, mais leur localisation reste encore inconnue.

\section{APPORTS ET LIMITES DU CROISEMENT DE DONNÉES}

Une grande continuité apparaît donc dans les méthodes employées par les archéologues depuis S. Müller et X. Nessel et reposant sur une lecture finalement assez intuitive du paysage : dans quelle mesure les outils informatiques nous permettent-ils aujourd'hui d'approcher ces questions sous 


\section{ArkeoGIS}

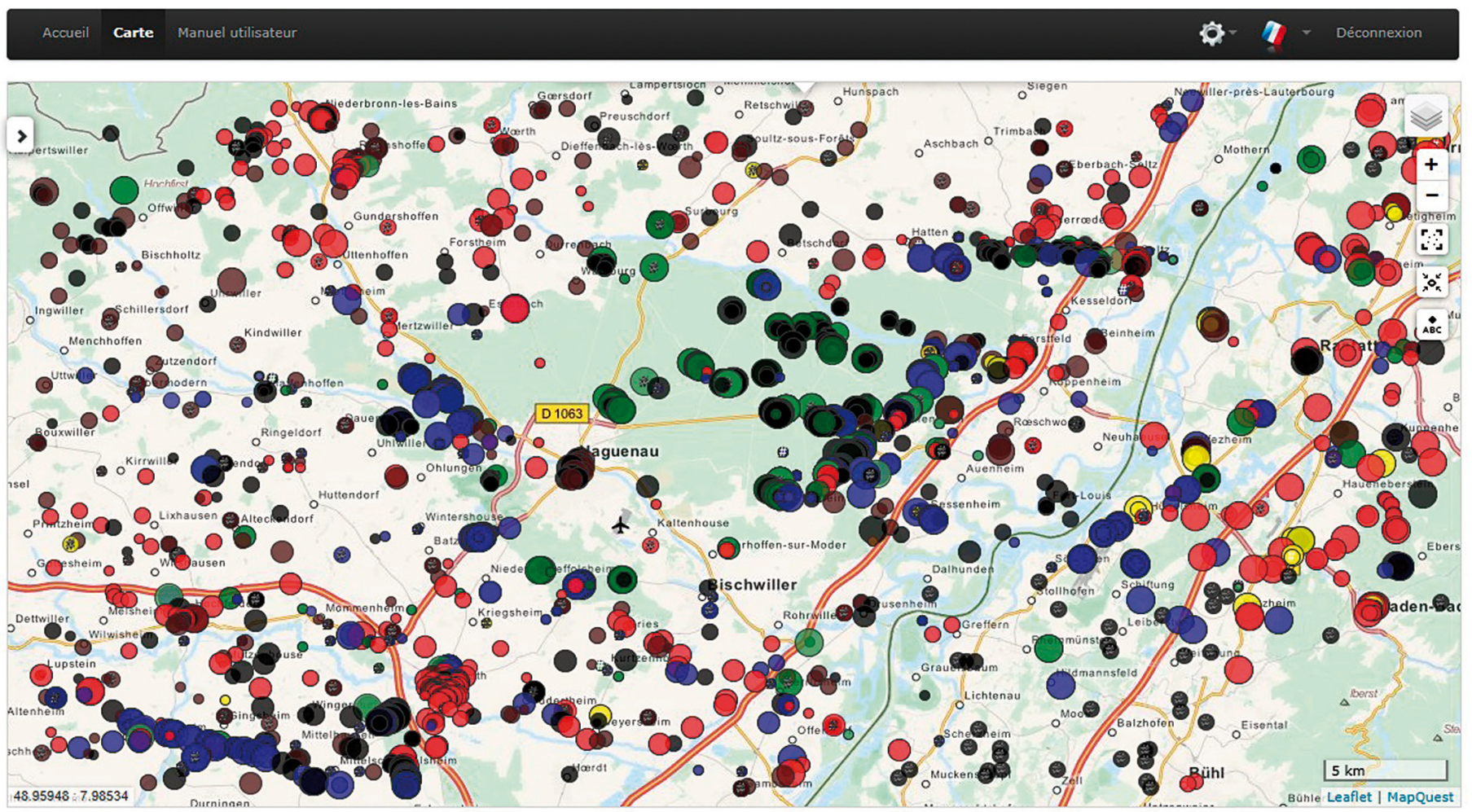

Récapitulatif des requêtes :

Requête \#1 $\bar{x}$

Figure 9

Ensembles des structures recensés.

un angle novateur ? Après avoir présenté certains résultats issus d'une agrégation de bases de données, nous proposerons des pistes d'analyse et des outils [17].

Le premier élément que permettent les SIG (Système d'Information Géographique) est de spatialiser précisément, sur différents fonds de cartes des informations données par des chercheurs ou des services de I'Inventaire. Ceci est facilité par des outils en ligne, dont ArkeoGIS. Initialement développé dans le cadre d'un financement INTERREG afin de mutualiser les données archéologiques et paléoenvironnementales de la vallée du Rhin, ArkeoGIS est un SIG bilingue (français-allemand), libre et en ligne. Actuellement dans sa troisième version, il permet de mettre en commun les données scientifiques disponibles sur une partie de la vallée du Rhin supérieur, certaines bases dépassant déjà largement ce cadre. Plusieurs dizaines de milliers de sites, objets et analyses sont listés pour le Rhin supérieur, et des fonds cartographiques ont été proposés afin que les utilisateurs puissent disposer de plusieurs jeux de cartes. Chaque auteur mettant à disposition des informations géoréférencées au format ArkeoGIS reste maître de celles-ci et peut seul décider de les modifier. Il peut très facilement accéder aux informations des autres contributeurs afin d'implémenter sa base. Un annuaire permet de mettre en contact les chercheurs, afin de développer les échanges, entre pays et entre institutions. L'outil offre donc l'interrogation en ligne de plusieurs dizaines de bases concernant la région des tumuli de Haguenau dans le cas présent. Douze bases issues de travaux de chercheurs représentant près de 2000 points auxquels s'ajoutent près de 1200 points issus de I'Inventaire français et allemand (ADABweb), ainsi qu'une centaine d'analyses et d'indices paléoenvironnementaux ou paysagers sont ainsi disponibles sur cette petite zone d'étude (fig. 9).

L'intégration de données précises - spatialement, chronologiquement, etc. - permet donc aux chercheurs d'avoir accès en quelques « clics » [18] à un affichage des sites présents autour de sa zone d'étude. Dans le cas des tumuli de la forêt de Haguenau, des données

[17] La bibliographie concernant ce type d'outils appliqués à l'archéologie est difficilement accessible dans la littérature archéologique. On conseillera le site en ligne www.archeogeographie.org/, une version papier ROBERT 2011 ou le manuel « information spatiale et archéologie », RODIER 2011.

[18] BeRNARD 2014. 
qui correspondaient encore il y a quelques années à plusieurs jours de dépouillement bibliographique sont désormais accessibles en ligne depuis n'importe quel support connecté. L'outil a grandement facilité la création et la mise en ligne d'une carte des tertres à l'échelle de I'Alsace [19].

L'utilisation d'ArkeoGIS a donc permis de mettre à disposition les données issues de nombreux travaux [20], productions françaises, allemandes et transfrontalières. Leur mise en ligne offre à l'utilisateur, pas nécessairement un spécialiste de SIG, un accès rapide à un état de la recherche, à de la littérature grise (rapports de fouille, études non publiées, travaux d'étudiants, etc.) et une réutilisation dans le logiciel de son choix.

Ces cartes vont nous permettre d'approcher avec une rapidité nouvelle de nombreuses questions pour lesquelles la documentation était jusqu'alors fastidieuse à obtenir, facilitant grandement la constitution de faisceaux d'indices sur ces questions. Les résultats de requêtes effectuées dans ArkeoGIS offrent une vision spatiale de I'information. En revanche, ce ne sont pas à proprement parler des cartes dans la mesure où il convient de reprendre les données, afin, en particulier, d'éliminer les doublons (certains sites étant en effet représentés dans plusieurs bases de données). Ce travail reste celui du chercheur mais, quoi qu'il en soit, il est bien plus rapide de fusionner des lignes dans un tableur géoréférencé que de poser des points manuellement sur des sujets dont on n'est pas forcément spécialiste.

Concernant l'étude des nécropoles tumulaires de la forêt de Haguenau, un certain nombre de questions peut être abordé en utilisant le SIG en ligne, au niveau de l'état de la recherche, des questions paléoenvironnementales et des analyses diachroniques.

Le premier effet immédiatement visible lors de la cartographie automatique [21] concerne l'état de la recherche (fig. 9). Des tracés linéaires correspondant, par exemple, aux études préliminaires à I'implantation de la LGV Est sont clairement lisibles au sud-ouest de la carte. Une zone vide importante se remarque au nord de Haguenau, dans la partie occidentale de la forêt actuelle. Ces vides sont intéressants, car ils peuvent permettre de modéliser

[19] Cette dernière est consultable sur le site en ligne de l'atlas historique d'Alsace. http://www. atlas. historique.alsace.uha.fr/ de-la-prehistoire-a-I-antiquite-tardive-von-der-urgeschichtebis-zur-spatantike/pat-culture/plonearticle.2014-0304.4439477114/.

[20] Voici les bases de données utilisées afin de produire les cartes. Elles proviennent d'ouvrages, de travaux d'étudiants, de chercheurs et des inventaires : ALISA (M. Trautmann), Bernard 2003, Engel 2013, Feliu 2008, Hoffmann 2007, Jacques 2014, Lasserre, Mischka 2007, Murgers (Schwartz), PAIR 2010, Roscio 2011, Wassong 2012, Schmid 2012, Schneider 2013, Tremblay-Cormier 2013, Wagner 2006, les densités de site, mais ils doivent être pondérés avant la production d'une carte définitive. En l'état, la carte fait clairement apparaître un déficit de sites d'habitat ou de production dans la forêt de Haguenau. En revanche, les sites importants, souvent perchés, qui se trouvent sur les versants orientaux du massif vosgien et occidentaux de la Forêt-Noire correspondent sans doute au débouché des axes de circulation dont il est question ici. Un approfondissement de ces problématiques est donc facilité par l'outil, I'accès immédiat aux données publiées et à la littérature grise (rapport de fouilles, travaux d'étudiants, analyses, etc.) est ici particulièrement appréciable. De même, le fait d'avoir un accès aux données des deux rives du fleuve de façon aisée et rapide est particulièrement utile. On regrettera évidemment de ne pas (encore) avoir accès aux données du Palatinat ou de Lorraine qui permettraient de reconsidérer l'importance de cols secondaires au sein des massifs.

En l'état des bases disponibles, I'utilisateur se rendra compte des limites intrinsèques à chaque base, dans I'Inventaire français (Patriarche) par exemple, les nécropoles de Haguenau sont des entités uniques (chaque tertre n'est donc pas référencé), alors que l'équivalent allemand (ADABweb) ne prend pas en compte les subdivisions chronologiques (Hallstatt/La Tène, tout étant de l'âge du Fer). L'accès à d'autres bases spécialisées, comme celle de L. Tremblay-Cormier sur les mobiliers métalliques, permet d'accéder à des données très précises (type d'objet, tombes exceptionnelles, etc.).

Ce type de bases permet d'avoir accès à des datations très précises, rares pour les nécropoles tumulaires et complexes à obtenir dans le cadre de travaux universitaires non publiés à ce jour.

En approfondissant encore les requêtes et en interrogeant les habitats, il apparaît rapidement un déficit de sites contemporains aux tertres, que ceux-ci n'aient pas été vus, qu'ils aient été érodés, recouverts ou simplement non encore référencés. Les données de datation précise sur les tertres complexifient I'approche ; en effet, seuls les tumuli fouillés pourront faire l'objet de tri par période, le reste devra être rattaché plus ou moins arbitrairement à I'une ou l'autre période. Des outils mathématiques

Wallanlagen (Morrissey 2012), Walter 2014 et Zehner habitats. Ces abréviations renvoient à des bases de données en ligne dont les descriptifs se trouvent donc sur le site d'ArkeoGIS. Un identifiant numérique pérenne et des métadonnées sont en cours de publication en ligne pour chacune.

[21] Sur toutes les cartes, la taille du symbole est indexée sur l'état de la recherche, la couleur correspond à la phase la plus récente du site : vert pour l'âge du Bronze, bleu pour l'âge du Fer, rouge pour la période romaine, jaune pour l'altomédiévale et grise pour les indéterminés. La présence de dièses sur les symboles correspond à des sites mal localisés placés sur le centroïde de la commune actuelle. 


\section{ArkeoGls \\ Le 18/02/2016 Loup Bernard peut consulter 64 Bases de données}

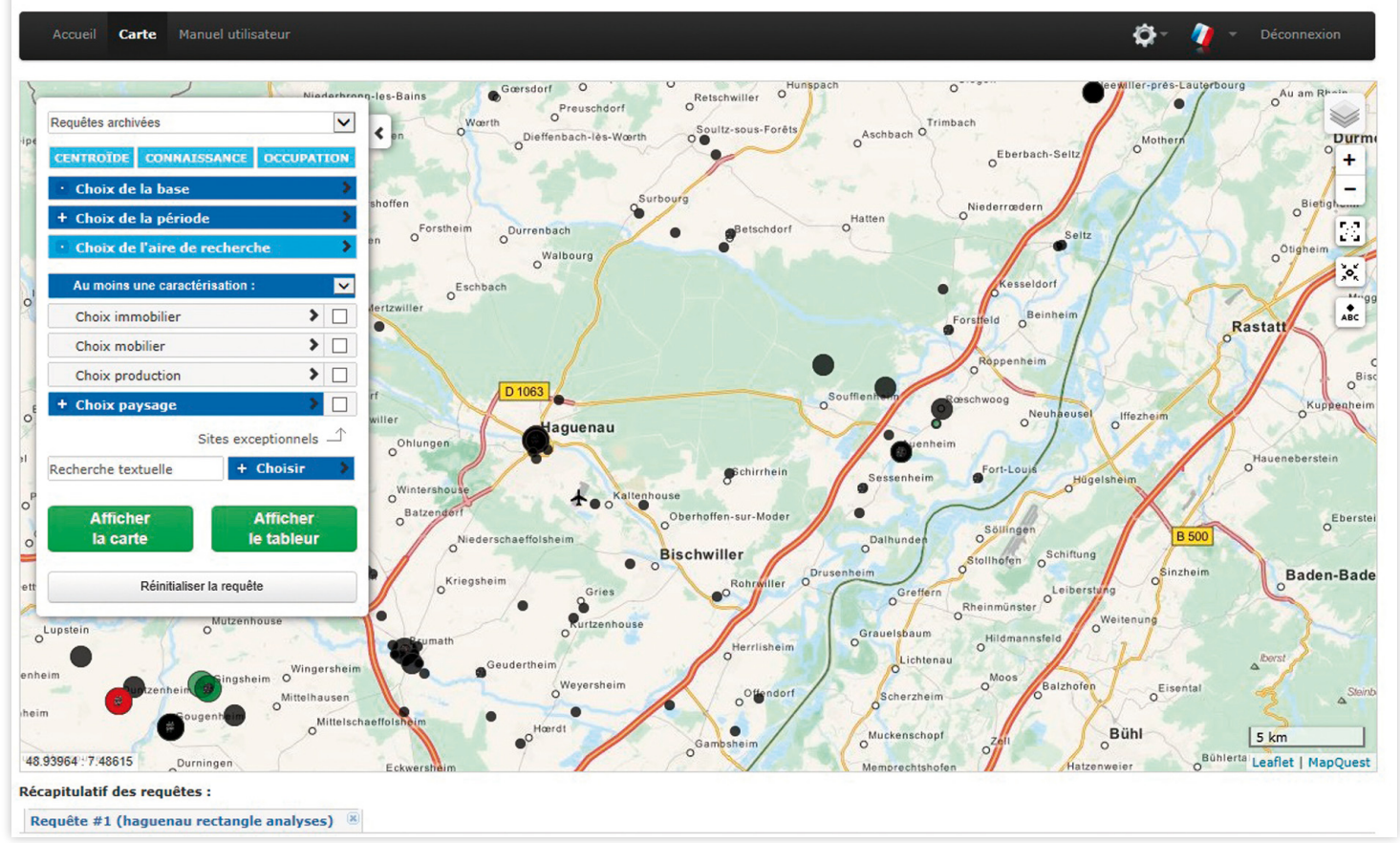

Figure 10

Analyses paléoenvironnementales et éléments de paysage. peuvent permettre de proposer des solutions à ce type d'interrogation, mais rien ne vaut les données de fouilles. Un accès rapide à l'état de la recherche transfrontalière est une nouveauté, alors qu'un tel état nécessitait jusqu'alors un fastidieux travail d'enquête dans les différents services de l'archéologie et chez les opérateurs de I'archéologie. Désormais l'agrégation dans ArkeoGIS, des travaux et de la mise à disposition de tout ou partie des informations relatives au site (coordonnées, datation, $n^{\circ}$ d'ordre, bibliographie) facilite au chercheur l'accès à l'état de la connaissance sans forcément une maîtrise de l'allemand dans le cas présent.

Les pleins et les vides de la carte peuvent se comprendre à l'aune de l'état de nos travaux archéologiques, mais peuvent également se révéler riches d'enseignement pour une approche paléoenvironnementale de la problématique. C'est le deuxième aspect que le SIG permet d'aborder (fig. 10). Le simple empilement des sites archéologiques le long du Rhin facilite par exemple l'identification des zones sur lesquelles tout ou partie des berges se sont stabilisées et leur époque de fixation (fig. 9). Selon la période d'occupation (âge du Bronze ou au Haut Moyen Âge), notre manière d'appréhender le paysage diffère, cette fonctionnalité - I'utilisation des gisements archéologiques comme fossiles directeurs pour l'étude de paléoméandres par exemple - est particulièrement appréciée des collègues géographes. Sans évidemment résoudre le détail de l'histoire de chaque paléochenal du fleuve (ce qui nécessiterait des centaines d'analyses [22]), I'outil offre du moins d'en dater certains à partir de fouilles ou d'études issues de données pédologiques ou environnementales.

Les liens entre différentes bases de données sont précieux : ainsi, ceux établis avec l'European Pollen Database permettent d'accéder à plus de 2000 taxons polliniques, la plupart étant en corrélation avec un $14 \mathrm{C}$ et une profondeur. Aucun n'est pour l'instant référencé autour de Haguenau, en revanche des points sont disponibles dans les Vosges et dans la Forêt-Noire, permettant par là un accès à de la littérature difficilement accessible pour un archéologue [23]. L'existence de $14 \mathrm{C}$ est également renseignée dans la thèse de $C$. Féliu et la base de M. Lasserre pour les sites de Gaentzenbruch et du Maimont.

ArkeoGIS liste également des travaux effectués par le laboratoire de pédologie de I'EOST [24] et par la géomorphologue de I'INRAP Grand Est Nord [25]. Certaines de ces études sont listées dans Patriarche (I'Inventaire français), mais pas leur intégralité. Patriarche ne liste pas non-plus les « diagnostics négatifs », un premier
[22] Voir par exemple Berendsen, Henk, Cohen et al. 2007. [23] RösCH 2012, renvoie à des pollens de La Tène ancienne qui ont été carottés à $60,5 \mathrm{~cm}$ de profondeur en Forêt Noire et $\mathrm{E}$. Gauthier pour le site de Hanau sans plus de précisions bibliographiques ou contextuelles (données issues de European pollen database).
[24] Base ALISA, renseignée par M. Trautmann, Laboratoire d'Analyses des Sols / LAS, UMS 830, plusieurs dizaines d'analyses renseignées.

[25] N. Schneider, géomorphologue et géoarchéologue INRAP, chercheure associée au laboratoire LIVE, UMR 7362, plusieurs dizaines d'analyses renseignées sur la zone. 
export a été fait [26] et montre un nombre important de tranchées exploratoires n'ayant livré aucun vestige dans la zone. Cette cartographie permet de rebondir sur des zones pour lesquelles le colluvionnement ou I'érosion peuvent expliquer I'absence de sites, travaux qui sont en cours de préparation avec I'EOST. Dans le cas où l'absence de sites ne s'expliquerait pas par des raisons taphonomiques, on pourra alors tester d'autres hypothèses, comme la présence de forêt par exemple, pour lesquelles les pollens seront un autre indice fort.

Comme tous les SIG, il est bien sûr possible de changer de fond cartographique pour afficher les points choisis. En plus des fonds libres de droits [27], le CIGAL permet d'avoir accès à un grand nombre de couches cartographiques dont certaines se révèlent très riches en enseignements [28]. Nous n'avons pas encore implémenté des fonds anciens de cartes, mais la possibilité de vérifier la présence de tertres sur différentes photos aériennes, dont le proche infrarouge et un modèle de terrain numérique composite ombragé rendent de grands services pour l'identification des tumuli les plus importants. Une base listant les zones actuelles à dominante humide, également mise à disposition par ce service, permet quant à elle d'identifier les zones de « rieds » actuels, dont beaucoup se superposent à des zones supposées ou attestées comme humides dans I'Antiquité.

Enfin, des programmes de recherche sur les parcellaires fossiles sont également contributeurs dans ArkeoGIS. En faisant une requête sur les termes «structures agraires », I'utilisateur a accès aux structures agraires listées dans l'inventaire français et allemand (crêtes de labours, champs bombés, etc.) ainsi qu'aux épierrements (murgers ou steinrudel) structurant le paysage depuis leur mise en place [29]. Ces éléments sont de bons indices quant aux ruptures et aux continuités dans le paysage (cf supra).

À partir de tous ces éléments, un chercheur dispose donc d'entrées documentaires précises pour aborder des questions paléo-environnementales : un accès à des publications en français et en allemand, une spatialisation de vestiges datés, le nom et le numéro des objets, sites archéologiques et analyses listés dans la zone ainsi que les coordonnées de la personne-relais sur chacune des bases. C'est par ce biais qu'il pourra être possible dans un avenir proche de déterminer si la forêt de Haguenau était présente lors de l'édification des tertres, si les nécropoles ont volontairement été placées dans des zones humides dans I'Antiquité ou si le cours des rivières et fleuves a évolué depuis les périodes concernées. Enfin, la possibilité facilitée de réinsuffler les sites choisis précisément dans un autre

[26] Nos remerciements à J.-P. Droux, ANARCHIS, UMR 7044 et G. Triantafillidis, SRA, pour avoir travaillé à la création de cet inventaire.

[27] Mapquest, openstreetmap, googlesat.

[28] Photos orthonormées IGN de 1997, 1998, 2002, 2011, scan IGN 2006 et 2010, photos proche infrarouge, MNT composite ombragé et base de données des zones à dominante humide. Le détail et le descriptif de chacun de ces fonds se trouvent sur le site du CIGAL : https://www.cigalsace.org/portail/.

[29] SchWARTZ et al. 2003.
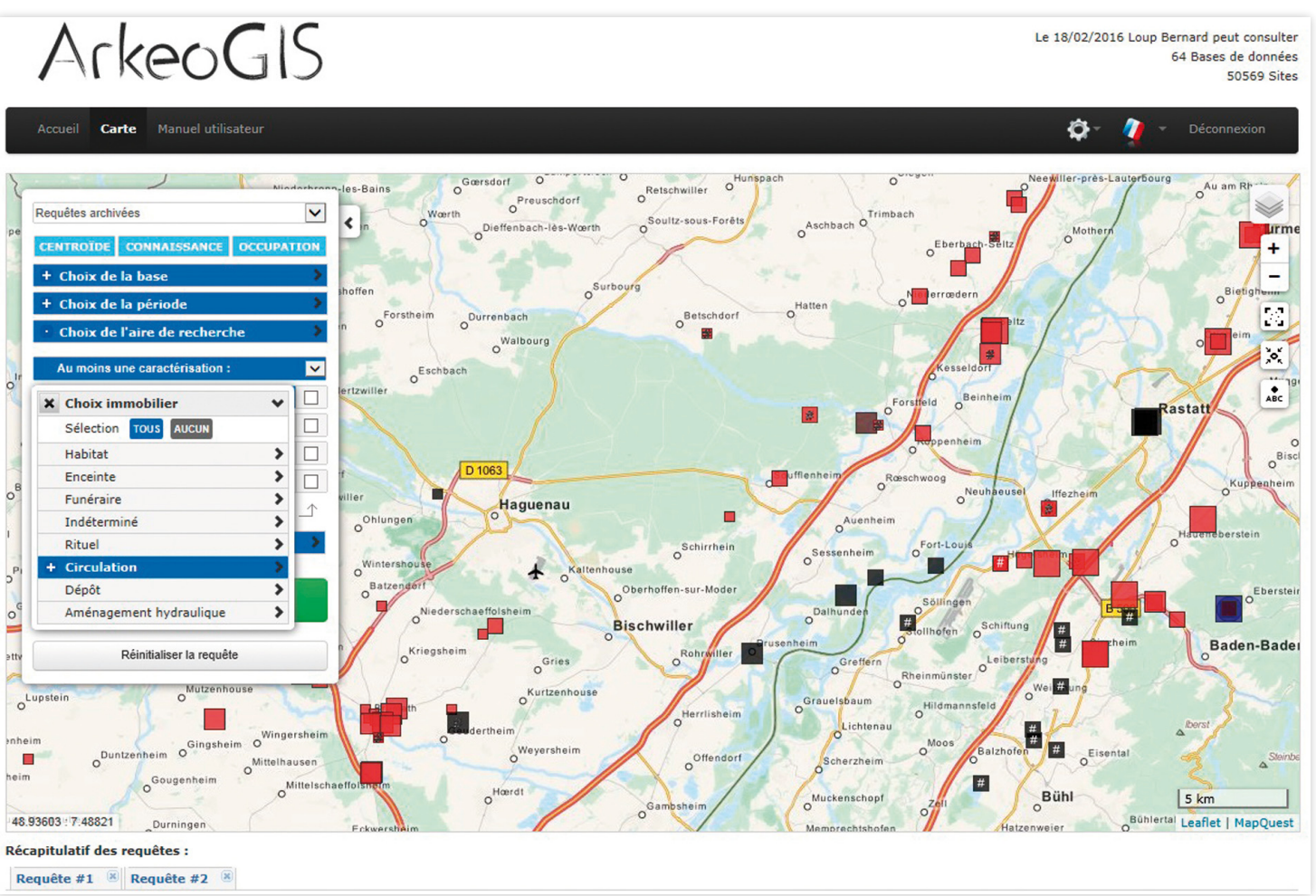

Figure 11

Voies et indices de voies (gués, bornes, ponts etc.) d'époques romaine (en rouge) et médiévale (en marron). 
SIG grâce à la fonction d'export est la garantie que les points correspondants aux sites et analyses seront bien placés sur la carte.

Une autre approche jusqu'alors fastidieuse se voit simplifiée par les outils agrégatifs : il s'agit de l'approche régressive diachronique. De nombreux travaux [30] utilisent ces méthodes, particulièrement sur les problématiques en relation avec les voies et les réseaux. Jusqu'alors, explorer méticuleusement l'intégralité des données archéologiques pour les deux rives du Rhin et ce en deux langues limitait largement ce type d'analyses. Grâce à ArkeoGIS, le chercheur peut obtenir immédiatement un état de la recherche pertinent de l'âge du Bronze au Haut Moyen Âge, les autres périodes étant en cours d'implémentation. On dispose d'informations directes (éléments de voirie romaine, bornes, stèles, ponts, cf. fig. 11), mais aussi indirectes. Ces dernières sont plus difficiles à expliciter, l'exemple le plus simple concernant les dépôts en milieu humide. Une première étude a déjà été citée (Logel 2007), une autre étude, plus complète et dont l'intégralité des données a pu être saisie dans ArkeoGIS [31], prend en compte près d'un millier de dépôts dans le cours du Rhin, entre l'âge du

[30] Voir, entre autres, Robert 2011, NAKoinz 2013, Delrieu \& MilCENT 2012, BOURGEOIS 2013 pour un aperçu récent sur le traitement des données dans le cadre des analyses de réseaux de communication et de la formation des paysages funéraires protohistoriques.

[31] SCHMID-MERKL 2012.

[32] Favory \& Van Der Leeuw 1998 p. 257-298.
Bronze et la période romaine. La juxtaposition de ces propositions et des tumuli (fig. 12) permet de disposer d'arguments plus solides sur des franchissements du fleuve que la méthode régressive se basant sur les gués médiévaux et modernes. Le fait qu'un utilisateur français puisse ainsi interroger et exploiter un travail de thèse allemand non encore publié est une nouveauté, et nous espérons que ce type de coopération pourra se renforcer dans les années à venir.

Un inventaire des axes principauxactuels - telsqu'ils sont cartographiés - et antiques - tels qu'ils transparaissent dans les différentes bases de données - est désormais facilement réalisable. L'utilisateur doit évidemment rester attentif aux modes de saisie différenciés des structures selon les pays, un tumulus prospecté apparaîtra « indéterminé » au niveau de la datation des bases françaises, alors que les Allemands proposeront une datation «âge du Bronze/Mérovingien » par exemple. Ainsi chaque utilisateur doit précisément réfléchir à la meilleure manière $d$ 'interroger les bases en fonction de leur contenu et des éléments qu'il maîtrise le mieux et le moins bien.

L'agrégation de sites et de bases met également en lumière des continuités et des ruptures. Moins efficace à ce titre que des projets de type Archaeomedes [32], travaillant directement sur les périodes de création et d'abandon, ArkeoGIS permet de poser ces questions en faisant des requêtes multiples. On voit, par exemple, bien se dessiner l'abandon des nécropoles tumulaires et d'une partie des voies déjà évoquées à la période romaine.
Figure 12

Voies, dépôts et tumuli recensés sur l'aire de recherche.

\section{ArkeoGls}

Le 18/02/2016 Loup Bernard peut consulter 64 Bases de données

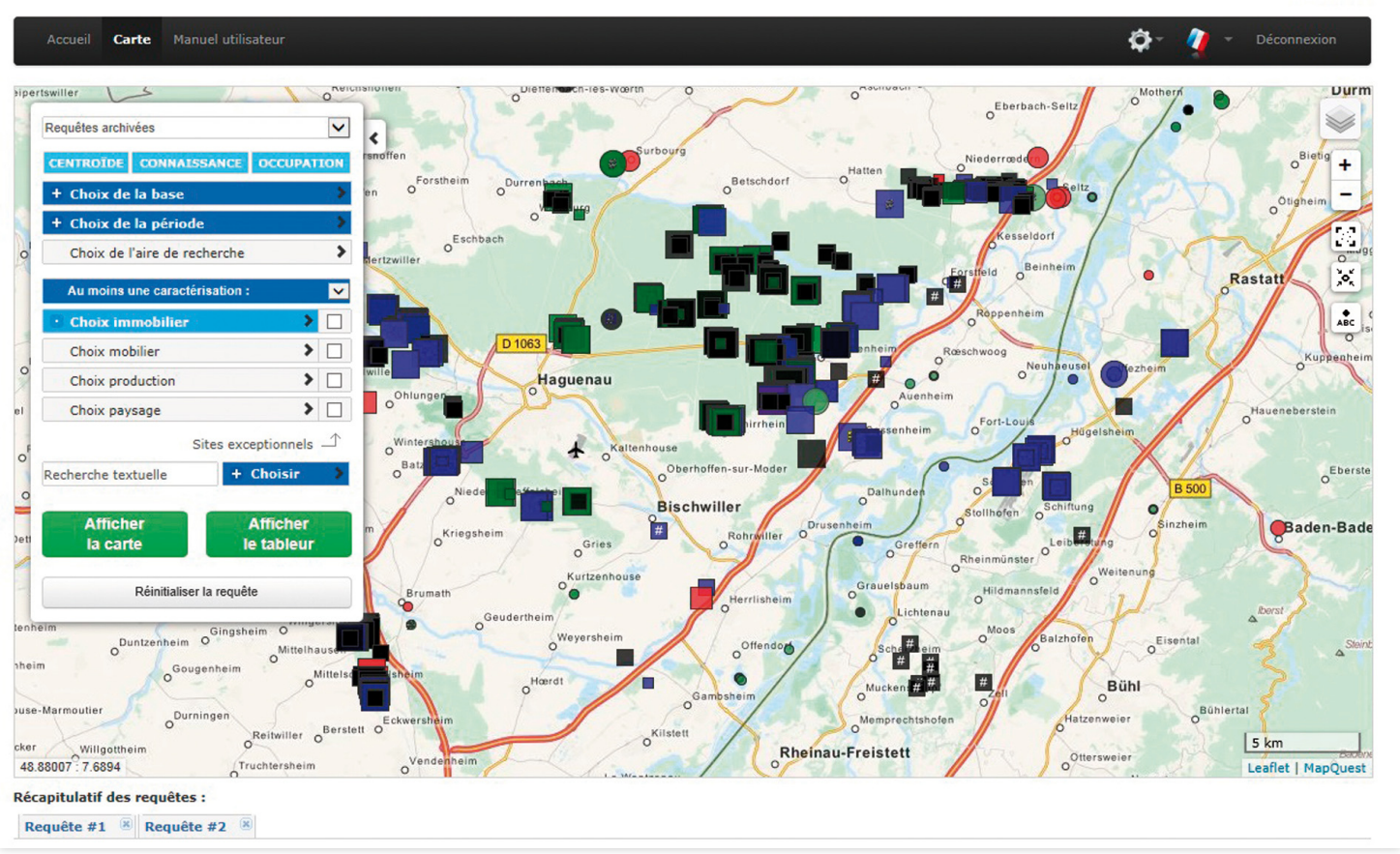




\section{OUVERTURES MÉTHODOLOGIQUES}

L'accès à de nombreuses données unifiées peut permettre, nous l'avons vu, d'appliquer plus facilement des outils statistiques pour traiter nos données. Cela a été fait par la SHKR [33] ou dans le cadre de la thèse de L. Tremblay-Cormier [34], I'exercice consistant à juxtaposer des marqueurs afin de déterminer respectivement des centralités théoriques ou des faciès. Ce type d'étude, qui est encore assez novateur en archéologie, permet de modéliser des relations et des faciès. Très théoriques et parfois ardues à suivre en détail, ces analyses deviennent plus intéressantes si les lecteurs peuvent modifier et implémenter les modèles eux-mêmes, en ayant accès aux bases de données source - ce qui est facilité par ArkeoGIS. Une fois encore, l'existence de plateformes agrégatives permet donc d'envisager avec plus d'efficacité des applications et des modèles avec un gain de temps bien supérieur au passé.

A minima, il est désormais possible de créer bien plus facilement des cartes de confiance, un outil permettant d'évaluer la représentativité et la validité de nos interprétations. À partir de ces cartes, il est possible de pondérer ensuite les propositions plus mathématiques et statistiques, créées par exemple à partir de systèmes multi-agents. Issu des recherches en intelligence artificielle, ce procédé consiste à attribuer des caractéristiques à des « agents » en fonction de leurs besoins et contraintes. La méthode a déjà été utilisée avec succès pour l'âge du Fer [35], son application aux problématiques en rapport avec les axes de circulation sous-entendrait un important travail préparatoire. Définir les villes, les lieux de production, de pouvoir et leur attractivité, ainsi que d'intégrer les types et vitesses de déplacement en fonction du Modèle Numérique de Terrain.

Tous ces outils sont « nourris » par des données qui doivent être hiérarchisées et homogénéisées. Une application de type ArkeoGIS permet d'implémenter les modèles et d'accélérer les transferts d'hypothèses régionales

[33] NAKOINZ 2013.

[34] TREMBLAY-CORMier (à paraître).

[35] Par exemple dans le projet CELTSIM, http:// celtsimulation. blogspot.fr/2014/08/celtsim-framework. html. en reproduisant dans d'autres secteurs d'étude des résultats existants. Il serait envisageable par exemple d'appliquer la méthode de regroupement proposée pour les ensembles funéraires normands (CHeValier 2009) à la zone de Haguenau. La possibilité de disposer d'un accès immédiat aux données facilitera ce genre d'échange de modèles dans un futur très proche.

Utiliser un agrégateur de sites facilite donc grandement la constitution d'un faisceau d'indices et permet de s'approcher de l'exhaustivité en fonction des bases disponibles dans I'outil. La spatialisation de la donnée permet également d'identifier des erreurs ou des concentrations spécifiques et de cibler des zones sur lesquelles des recherches seraient souhaitables. C'est aussi une approche obligeant à normaliser les données : une fois cette normalisation effectuée, l'information peut servir dans d'autres cas. Toute requête effectuée à l'aide d'ArkeoGIS permet d'obtenir le tableau correspondant, au format.csv [36]. En somme, la mise en ligne de bases de données est une nouvelle forme de publication, à la différence du papier, les données interagissent entre elles au sein de l'outil, pour peu qu'elles aient une caractérisation commune (chronologie, structures, mobiliers, emplacement, etc.). Cette transformation du format de publication n'est que le début d'un glissement de nos outils vers une plus grande utilisation des bases de données afin de justifier nos hypothèses.

Au final, après plus d'un siècle d'études sur les voies, et malgré le développement d'outils de plus en plus puissants, comparatismes, cartes anciennes et pointillés restent d'actualité pour une archéologie des réseaux et ils risquent de le rester encore quelque temps. La facilité d'obtention de l'information préparatoire à un travail de cartographie automatique à l'aide d'un SIG est désormais telle que le développement d'analyses systématiques à différentes échelles se développera rapidement dans les années à venir.

[36] Comma Separated Value, un format universel utilisable par tout autre logiciel de type tableur (excel etc.), base de données (de type Access ou Filemaker), SIG (QGIS, ArcGIS, par exemple), mais aussi vers des outils plus complexes de statistiques ou de graphes. 
AberT, Franck, 2009, Rapport de prospection, Haguenau (Bas-Rhin), Kurzgelaend, Schirrheinerweg, PAIR.

Abert, Franck \& Rieth, Pascal, 2010, Rapport de prospection, Brumath (Bas-Rhin), Forêt de Brumath, PAIR.

Berendsen, Henk, Cohen Kim et al. 2007, « The use of GIS in reconstructing the Holocene palaeogeography of the Rhine-Meuse delta », The NetherlandsInternational Journal of Geographical Information Science 21, p. 589-602.

Bernard, Loup, 2014, « ArkeoGIS v2.0, Eléments d'analyse de la mise en ligne de bases multilingues sur fond cartographique : fonctionnalités, apports et limites » dans Laurent Costa et al. (éd.) Actes des 3èmes Journées d'Informatique et Archéologie de Paris, JIAP 2012 (Archeologia e Calcolatori supplemento 5), p. 228-237.

Bernard, Loup, Ertlen, Damien \& Schwartz, Dominique, 2015, « ArkeoGIS, merging Georgraphical and Archaeological datas online », dans François Giligny et al. (éd.), Concepts, methods and tools. Proceedings of the 42nd Annual Conference on Computer Applications and Quantitative Methods in Archaeology, Oxford.

Bourgeois, Quentin, 2013, Monuments on the Horizon, the formation of the barrow landscape throughout the 3rd and 2nd Millenium $B C$, Leiden.

Chevalier, Arnaud, 2009, «Espaces funéraires et réseaux viaires protohistoriques en Basse-Normandie », Les nouvelles de l'archéologie, 115, p. 44-51.

Delrieu, Fabien \& Milcent, Pierre-Yves, 2012, « Les paysages tumulaires protohistoriques dans le Massif Central : les exemples du Cézallier et du Causse Noir », dans Daniel Bérenger, Jean Bourgeois, Marc Talon \& Stefan Wirth (éd.), Gräberlandschaften der Bronzezeit - Paysages funéraires de l'âge du Bronze, Actes du Colloque APRAB/LWL de Herne (Westphalie, Allemagne) du 15 au 18 octobre 2008, Darmstadt, p. 23-57.

Dumont, Anne, 2002, Les passages à gué de la Grande Saône : approche archéologique et historique d'un espace fluvial (de Verdun-sur-le-Doubs à Lyon), Revue archéologique de l'Est, supplément 17, Dijon.

Favory, François \& VAN Der Leeuw, Sander, 1998, « Archaeomedes, la dynamique spatio-temporelle de l'habitat antique dans la vallée du Rhône : bilan et perspectives. », Revue archéologique de Narbonnaise 31, pp. 257-298.

Frölund, Per, 2009, En skärvstenhög i Gamla Uppsala, Arkeologisk undersökning, Gamla Uppasala, Fornlämning 119, Uppsala sn, Uppland, rapporter 2009:07, Uppsala.

Kiмmig, Wolfgang, 1983, Die Heuneburg an der oberen Donau, Stuttgart.

KLASSEN, Lutz, 2014, Along the road. Aspects of causewayed enclosures in south Scandinavia and Beyond, Arhus.

LegendRe, Jean-Pierre, 1989, «Organisation spatiale et "pouvoir princier" dans la région de Haguenau (Bas-Rhin) à la fin de l'époque Hallstattienne », Revue archéologique de l'Est 40, p. 199-203.

LJUNGKvist, John, 2013, « Monumentaliseringen av Uppsala », dans Olof Sundqvist et Per Vikstrand avec la collaboration de John Ljunkvist (éd.), Gamla Uppsala i ny belysning, Uppsala p. 33-68.

Logel, Thierry, 2007, «Les dépôts de métal en milieu humide et les gués sur le Rhin et l'Ill à l'âge du Bronze : présentation préliminaire », dans Cahiers Alsaciens d'Archéologie, d'Art et d'Histoire 50, p. 9-17.

MüLleR, Sophus, 1897, Vor oldtid. Danmarks forhistoriske archæologi, København.

Müller, Sophus, 1904, « Vej og bygd i sten- og bronzealderen », Arbøger for nordisk Oldkyndighed og Historie, p. 1-64.

NakoInz, Oliver, 2013, Archäologische Kulturgeographie der ältereisenzeitlichen Zentralorte Südwestdeutschlands, Bonn (Universitätsforsch. prähist. Arch. 224).

Naue, August Wilhelm, 1905, Die Denkmäler der vorrömischen Metallzeit im Elsass, Strassburg.

Robert, Sandrine (éd.) 2011, Sources et techniques de l'archéogéographie, Besançon.

RodieR, Xavier (éd.), 2011, Information spatiale et archéologie, Paris.

Rösch, Manfred, 2012, «Vegetation und Waldnutzung im Nordschwarzwald während sechs Jahrtausenden anhand von Profundalkernen aus dem Herrenwieser See », Standort.wald 47, p. 43-64.

Schaeffer, Claude, 1926, Les tertres funéraires préhistoriques dans la forêt de Haguenau, I, les Tumulus de l'âge du Bronze, Haguenau.

Schaeffer, Claude, 1930, Les tertres funéraires préhistoriques dans la forêt de Haguenau, II, les Tumulus de l'âge du Fer, Haguenau.

Schaeffer, Claude, 1982, Les tertres funéraires préhistoriques dans la forêt de Haguenau, III, le carnet de fouille de $X$. J. Nessel, Haguenau.

Schmid-MerkL, Christiane, 2012, Der Oberrhein - Archäologie einer metallzeitlichen Flusslandschaft, thèse sous la direction de Christoph Huth, Freiburg i. Breisgau.

Schwartz, Dominique, Bernardy, Laurence, Jouve-Litaudon, Claire \& Vonna, Anne, 2003, « Le Bickenberg à Osenbach (Haut-Rhin) 》, Revue Géographique de l'Est 43 (3).

Tremblay Cormier, Laurie (à paraître), Identités culturelles et échanges entre Rhin et Rhône du $x^{e}$ au $v^{e}$ siècle avant notre ère, Dijon.

WAssong, Rémy, 2013, « Pourquoi le tumulus? Les tumuli celtiques à la lumière des sagas scandinaves, essai de comparaison », Histoire antique et médiévale 70, p. 64-69.

WAssong, Rémy, 2015, «Tumulus et milieux humides: rites, ostentation et lieux de passages. Les tertres de l'âge du Fer dans le fossé rhénan », dans Fabienne Olmer \& Réjane Roure (éd.), Les Gaulois au fil de l'eau, Actes du $37^{e}$ colloque international de I'AFEAF, Montpellier, 8-11 mai 2013, Bordeaux, p. 1153-1162. 